\title{
The socioeconomic aspects of nonalcoholic fatty liver disease: food insecurity as a novel risk factor for steatosis and liver fibrosis
}

\author{
Josephine Frohme, Frank Tacke \\ Department of Hepatology \& Gastroenterology, Charité University Medicine Berlin, Berlin, Germany \\ Correspondence to: Prof. Frank Tacke, MD, PhD. Chairman and Professor of Medicine, Charité Universitätsmedizin Berlin, Department of \\ Hepatology \& Gastroenterology, Augustenburger Platz 1, D-13353 Berlin, Germany. Email: frank.tacke@charite.de. \\ Provenance and Peer Review: This article was commissioned by the editorial office, Hepatobiliary Surgery and Nutrition. The article did not undergo \\ external peer review. \\ Comment on: Golovaty I, Tien PC, Price JC, et al. Food Insecurity May Be an Independent Risk Factor Associated with Nonalcoholic Fatty Liver \\ Disease among Low-Income Adults in the United States. J Nutr 2020;150:91-8.
}

Submitted Feb 10, 2020. Accepted for publication Feb 24, 2020.

doi: 10.21037/hbsn.2020.03.03

View this article at: http://dx.doi.org/10.21037/hbsn.2020.03.03

Over the last decade, the global prevalence of nonalcoholic fatty liver disease (NAFLD) in both adults and children was rapidly increasing and has become the most common cause of chronic liver disease in many parts of the world. It is predicted to become also the most frequent indication for liver transplantation by 2030 and fueling the rising incidence and prevalence of primary liver cancer in western countries $(1,2)$.

Food insecurity is defined as the limited or uncertain availability of nutritionally adequate and safe foods or limited or uncertain ability to acquire acceptable foods in socially acceptable ways' (3). The US Department of Agriculture has developed tools to assess the severity of food insecurity. A questionnaire consisting of 18 items can be used to divide the severity of food insecurity into four areas. Subjects reporting "low" or "very low" food security can be considered "food insecure".

In their recent study published in the January 2020 issue of the Fournal of Nutrition, Golovaty and coworkers hypothesized that food insecurity would be associated with an increased risk of NAFLD among adults in low-income households. While associations between food insecurity and cardiometabolic risk at the one hand, and cardiometabolic risk factors and NAFLD on the other hand are well reported (2), the study by Golovaty I and coworkers is the first study that examined the (potentially direct) association of food insecurity with NAFLD (Figure 1) (5).

The study analyzed data from the National Health and
Nutrition Examination Survey (NHANES), a complex multistage, stratified, clustered probability sample collected in the US between 2005 and 2014 from cross-sectional surveys, investigations and laboratory data. In models adjusted for demographic, socioeconomic, and behavioral health characteristics, food insecurity was associated with higher odds of estimates of NAFLD [adjusted odds ratio (AOR): 1.38; 95\% confidence interval (CI): 1.08, 1.77), advanced fibrosis (AOR: 2.20; 95\% CI: 1.27, 3.82), obesity (AOR: 1.32; 95\% CI: 1.06, 1.66) and diabetes (AOR: 1.41; $95 \%$ CI: $0.999,1.982, \mathrm{P}=0.05)$ compared with living in a food-secure household (5).

In this correlative analysis, the occurrence of NAFLD was determined using the US Fatty Liver Index (USFLI), while advanced liver fibrosis was estimated using the NAFLD Fibrosis Score. Both endpoints were determined using scoring systems based on ethnic and laboratory data. Unfortunately, no histopathological or morphological criteria were used for the diagnosis. In principle, liver biopsy is recommended as the gold standard method for the diagnosis and staging of fibrosis in patients with NAFLD as well as other chronic liver diseases (3). However, this procedure is associated with a risk of complications, is costly, time-consuming and it is therefore deemed logistically impossible to enforce liver biopsy in all patients with NAFLD. Therefore, the observed association with food insecurity and NAFLD or fibrosis should be verified in an independent cohort with histologically diagnosed 


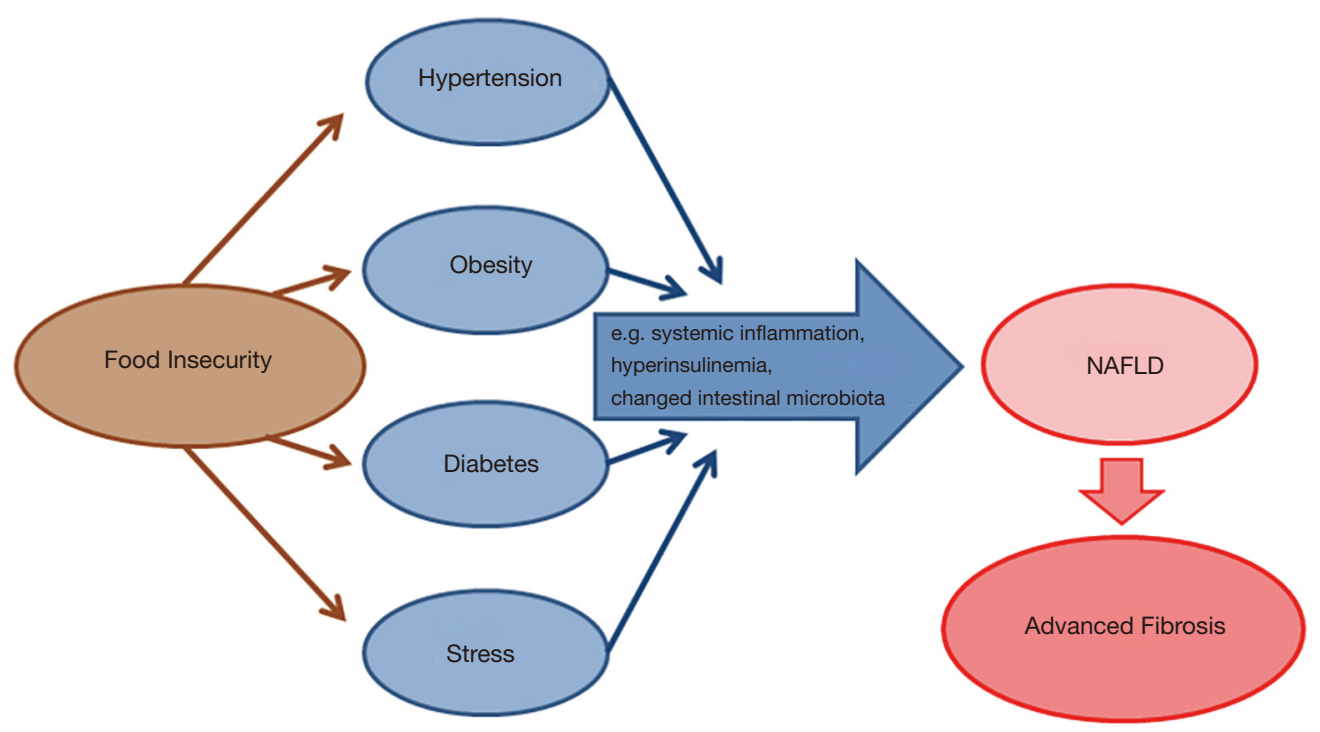

Figure 1 Food insecurity is associated with obesity, diabetes and hypertension. It is predicated on both financial instability and a sense of anxiety surrounding access to healthy food with results in a higher level of stress (4). Food insecurity drives a cardiometabolic risk profile, which is a risk factor for hepatic fat and hepatic fibrosis development in NAFLD (1). The study by Golovaty and coworkers for the first time demonstrate an independent association between food insecurity and NAFLD. Possible mechanisms of this relationship could be, for example, systemic inflammation, hyperinsulinemia and changes in the intestinal microbiota $(1,2,5,6)$.

and staged fatty liver disease in the future. Histological data would potentially also allow to link food insecurity with disease activity, e.g., the presence on non-alcoholic steatohepatitis (NASH).

Furthermore, the reported outcomes (NAFLD, fibrosis) are surrogate markers, and the study did not investigate mortality as an endpoint. While the NAFLD Fibrosis Score has been widely evaluated in other studies for its diagnostic effectiveness, the score is considered particularly useful for excluding advanced fibrosis (stage $\geq 3$ ) and potentially also for predicting liver-related mortality (7). Future analyses from the NHANES cohort should assess potential associations between food insecurity, NAFLD/ fibrosis and 'patient-relevant outcomes' (i.e., risk of liver cirrhosis, decompensation, hepatocellular carcinoma, liver transplantation, and death).

Of note, comparing food-secure with food-insecure adults, the unadjusted, estimated NAFLD prevalence and unadjusted, estimated advanced fibrosis prevalence did not significantly differ, emphasizing the great importance of confounders for assessing such associations. All criteria of food insecurity as well as the behavioral health characteristics like daily alcohol intake or smoking consumption are based on self-reported questionnaires.
Self-reporting data can be affected by an external bias caused by social desirability or approval, especially in cases where anonymity and confidentiality cannot be guaranteed at the time of data collection (8). Nevertheless, this study was the first to investigate the relationship between economic and political factors in the development of NAFLD and its associated diseases in a large sample size of patients.

At the same time, this study poses the interesting question, whether food insecurity exacerbates known metabolic risk factors as an effect modification or as a true causal relation and directly drives liver injury. Previous studies have already shown that food insecurity as a risk factor for metabolic diseases has both a direct effect on the control of blood sugar levels and an indirect effect on selfsufficiency through stress (4). Since cardiometabolic diseases and NAFLD share the same risk factors, the association between food insecurity and NAFLD is not unexpected. Moreover, as food insecurity is a risk factor in the pathogenesis of diabetes by influencing the blood glucose level (4), the pathogenic influence in the development of NAFLD by hyperinsulinemia is conceivable (2).

Based on the U.S. Household Food Security Survey Module, food insecurity is predicated on both financial 
instability and a sense of anxiety surrounding access to healthy food (3). In addition to unhealthy dietary habits, previous studies have already shown that food insecurity leads to increased stress caused by insufficient access to food. This leads to an increased cortisol production with a consecutive increased level of mediators of systemic inflammation (6). These mechanisms of systemic inflammatory response are involved in the pathogenesis of chronic diseases such as NAFLD (2). The results thereby suggest that food insecurity may directly influence health outcomes through specific biological mechanisms.

In addition, food insecurity could influence the composition of the gut flora by reducing the quality and composition of the food. In the past, intestinal microbiota has been shown to influence the susceptibility of the host to obesity, liver steatosis, non-alcoholic steatohepatitis, liver fibrosis and primary liver cancer. Conversely, host factors influence the intestinal microbiota, such as food composition and obesity (2).

In conclusion, the study by Golovaty and coworkers for the first time demonstrate an independent association between socio-economic factors and NAFLD. While the underlying mechanisms on how these socio-economic factors influence biological processes and thus contribute to the development of NAFLD disease remain to be determined, the social conditions must be considered as part of the multifactorial pathogenesis of NAFLD. These findings shed light on a neglected area of metabolic liver diseases and might be used in disease prevention strategies that require both health policy and medical resources (9).

\section{Acknowledgments}

Funding: None.

\section{Footnote}

Conflicts of Interest: Both authors have completed the ICMJE uniform disclosure form (available at http://dx.doi. org/10.21037/hbsn.2020.03.03). FT serves as an unpaid editorial board member of HBSN. The other author has no conflicts of interest to declare.

Ethical Statement: The authors are accountable for all aspects of the work in ensuring that questions related to the accuracy or integrity of any part of the work are appropriately investigated and resolved.
Open Access Statement: This is an Open Access article distributed in accordance with the Creative Commons Attribution-NonCommercial-NoDerivs 4.0 International License (CC BY-NC-ND 4.0), which permits the noncommercial replication and distribution of the article with the strict proviso that no changes or edits are made and the original work is properly cited (including links to both the formal publication through the relevant DOI and the license). See: https://creativecommons.org/licenses/by-nc-nd/4.0/.

\section{References}

1. Byrne CD, Targher G. NAFLD: a multisystem disease. J Hepatol 2015;62:S47-64.

2. Diehl AM, Day C. Cause, Pathogenesis, and Treatment of Nonalcoholic Steatohepatitis. N Engl J Med 2017;377:2063-72.

3. Heerman WJ, Wallston KA, Osborn CY, et al. Food insecurity is associated with diabetes self-care behaviours and glycaemic control. Diabet Med 2016;33:844-50.

4. Walker RJ, Williams JS, Egede LE. Pathways between food insecurity and glycaemic control in individuals with type 2 diabetes. Public Health Nutr 2018;21:3237-44.

5. Golovaty I, Tien PC, Price JC, et al. Food Insecurity May Be an Independent Risk Factor Associated with Nonalcoholic Fatty Liver Disease among Low-Income Adults in the United States. J Nutr 2020;150:91-8.

6. Gowda C, Hadley C, Aiello AE. The association between food insecurity and inflammation in the US adult population. Am J Public Health 2012;102:1579-86.

7. Yoneda M, Imajo K, Takahashi H, et al. Clinical strategy of diagnosing and following patients with nonalcoholic fatty liver disease based on invasive and noninvasive methods. J Gastroenterol 2018;53:181-96.

8. Althubaiti A. Information bias in health research: definition, pitfalls, and adjustment methods. J Multidiscip Healthc 2016;9:211-7.

9. Lazarus JV, Ekstedt M, Marchesini G, et al. A cross-sectional study of the public health response to non-alcoholic fatty liver disease in Europe. J Hepatol 2020;72:14-24.

Cite this article as: Frohme J, Tacke F. The socioeconomic aspects of nonalcoholic fatty liver disease: food insecurity as a novel risk factor for steatosis and liver fibrosis. HepatoBiliary Surg Nutr 2020;9(4):543-545. doi: 10.21037/hbsn.2020.03.03 